\title{
Caracterização das habilidades simbólicas de crianças com síndrome de Down
}

\author{
Characterization of the symbolic abilities of children with \\ Down syndrome
}

\author{
Mariane Nardezi Ciciliato ${ }^{1}$, Daiana Camargo Zilotti², Patrícia Pupin Mandrá ${ }^{3}$
}

\begin{abstract}
RESUMO
Objetivo: Caracterizar as habilidades simbólicas de um grupo de crianças com síndrome de Down. Métodos: Participaram do estudo 26 crianças com idades entre 12 e 36 meses, divididas em dois grupos: grupo síndrome de Down (GSD) e grupo controle (GC) - crianças com desenvolvimento normal. Os grupos foram subdivididos de acordo com a idade: GSD I e GC I, compostos por crianças de 12 a 24 meses; GSD II e GC II, com crianças de 25 a 36 meses. Os dados foram coletados por meio da interação com a examinadora em situação lúdica, durante 30 minutos o GSD e 20 minutos o GC, de acordo com a proposta do protocolo de observação comportamental. Resultados: Comparando ambos os grupos controle encontramos diferença $(\mathrm{p}<0,05)$ para as formas de manipulação dos objetos, para o nível de desenvolvimento do simbolismo e para o desempenho geral no protocolo. Em ambos os grupos síndrome de Down houve diferença para o nível de desenvolvimento do simbolismo. Na comparação inter-grupos de acordo com as faixas etárias encontramos diferenças quanto à forma de manipulação dos objetos, nível de desenvolvimento do simbolismo e desempenho geral no protocolo. A imitação sonora e gestual não se diferenciou significativamente nessa pesquisa. Conclusão: Os resultados confirmaram a hipótese de atraso do desenvolvimento das habilidades simbólicas para crianças com síndrome de Down. $\mathrm{O}$ exame da linguagem e simbolismo em contexto funcional possibilitou a confrontação das manifestações observadas neste grupo e as descritas para crianças com desenvolvimento linguístico e simbólico considerados normais, sendo o nível de desenvolvimento simbólico o melhor parâmetro de análise e acompanhamento para o grupo.
\end{abstract}

Descritores: Síndrome de Down; Linguagem infantil; Simbolismo; Desenvolvimento infantil; Cognição

\section{INTRODUÇÃO}

A síndrome de Down (SD) é uma das síndromes mais conhecidas e estudadas. Suas características foram primeiramente descritas por John Langdon Down em 1866. A SD é a principal causa genética da deficiência mental (DM), e é resultante da presença extra de um braço longo do cromossomo 21, caracterizando trissomia do 21 . O excesso de material genético pode ocorrer de três maneiras diferentes: por trissomia

Trabalho realizado no Curso de Fonoaudiologia da Faculdade de Medicina de Ribeirão Preto da Universidade de São Paulo - USP - Ribeirão Preto (SP), Brasil.

(1) Fonoaudióloga da Equilíbrio Escola de Educação Especial mantida pela Associação de Pais e Amigos dos Excepcionais - APAE - Tambaú (SP), Brasil. (2) Fonoaudióloga do Hospital Neurológico Ritinha Prates - Araçatuba (SP), Brasil.

(3) Doutora, Professora do Curso de Fonoaudiologia do Departamento de Oftalmologia, Otorrinolaringologia e Cirurgia de Cabeça e Pescoço da Faculdade de Medicina de Ribeirão Preto da Universidade de São Paulo - USP - Ribeirão Preto (SP), Brasil.

Endereço para correspondência: Mariane Nardezi Ciciliato. R. João Godoy, 307, Centro, Tambaú (SP), Brasil, CEP: 13710-000. E-mail: marianeciciliato@yahoo.com.br

Recebido em: 5/8/2009; Aceito em: 29/1/2010 simples ou não disjunção do cromossomo 21, resultando num cariótipo constituído por 47 cromossomos; por translocação, quando o cromossomo 21 extra está ligado a outro cromossomo constituindo um cariótipo com 46 cromossomos; e por mosaicismo, caracterizado pela ocorrência de linhagem celular normal e trissômica ${ }^{(1-3)}$.

Os aspectos clínicos mais frequentes na SD são: comprometimento intelectual; hipotonia muscular; fissura palpebral oblíqua; aumento da vascularização retiliana; occipito achatado; hiperextensão articular; mãos largas e dedos curtos; baixa estatura; cardiopatias congênitas, clinodactilia do quinto dedo; orelhas de implantação baixa; orelhas displásicas; epicanto; prega palmar única transversa; instabilidade atlantoaxial; e instabilidade rótulo-femural; braquicefalia; base nasal achatada; hipoplasia da região mediana da face; língua protrusa e hipotônica; retardo no desenvolvimento das funções auditivas e visuais; predominância por respiração oral; pescoço curto e grosso; e sonolência ${ }^{(1-3)}$.

A ocorrência desta síndrome não tem preferência por gênero ou raça. A idade materna maior que 30 anos é relatada como um dos fatores de risco, mas não exclui a possibilidade do pai contribuir para a ocorrência ${ }^{(3-4)}$. 
Geralmente, a família estimula a criança com síndrome de Down desde o nascimento e esta é uma condição favorável para a aquisição de habilidades nos mesmos intervalos de tempo observados em crianças normais. Deve-se atentar para que os cuidados sejam equilibrados, de modo a incentivar a independência dessas crianças e evitar que cuidadores as substituam em tarefas que poderiam ser desempenhadas por elas ${ }^{(5,6)}$. Mães de crianças com de síndrome de Down podem usar mais ajuda física por achar que o filho não é capaz de realizar algumas atividades sozinho ${ }^{(7)}$.

Desde os primeiros meses, a criança com SD tem dificuldade de manter a atenção e de estar alerta aos estímulos externos. Em geral, são menos interativas e respondem menos ao adulto, mas isso não significa que não sejam capazes de desenvolver a atenção e interação, embora isso ocorra de forma e em momentos diferentes quando comparados à criança sem atraso ${ }^{(8)}$.

As crianças com SD apresentam atrasos significativos de linguagem ${ }^{(9)}$. As habilidades são adquiridas na mesma sequência observada em crianças normais, porém com defasagem cronológica $^{(10-12)}$.

O desenvolvimento da linguagem oral é correlacionado com uma série complexa de habilidades cognitivas, perceptuais e linguísticas cuja gênese está no período pré-verbal. A construção simbólica faz parte das habilidades cognitivas essenciais para a formação do signo linguístico e consequentemente da utilização de vocábulos como forma de expressão. Assim, o desenvolvimento do simbolismo está diretamente relacionado ao da linguagem oral.

A formação progressiva das condutas presentes na brincadeira simbólica inicia-se com as habilidades pré-simbólicas que são: uso convencional de objetos; esquemas simbólicos; início da aplicação das ações em pessoas e bonecos. Seguese condutas simbólicas, a saber: sistematização da aplicação das ações em outros; sequencialização das ações simbólicas; e uso de símbolos ${ }^{(13,14)}$.

A atividade simbólica pode ser compreendida como a representação das experiências, capacidade para abstrair as informações provenientes das ações praticadas desde o nascimento, e que mudam com o decorrer dos anos ${ }^{(15)}$.

Em relação à comunicação, a linguagem não-verbal é um dos meios mais utilizados por crianças com SD. Normalmente, elas preferem os gestos à produção verbal, sendo os gestos dêiticos (dar, mostrar, apontar) e os gestos representativos (levar telefone à orelha, colher na boca) produzidos com maior frequência ${ }^{(6,16)}$. Muitas vezes, começam com uma comunicação predominantemente gestual e, a seguir, evoluem para a linguagem oral e comunicação gestual simultâneas ou para uma maior variação na comunicação gestual ${ }^{(17)}$.

Explorações de processos de aquisição de relações simbólicas têm importantes implicações para ampliar a compreensão da aquisição de linguagem, no que concerne, por exemplo, ao desenvolvimento de repertórios de nomeação, uso de sinônimos, e organização hierárquica de categorias ${ }^{(18)}$.

A SD é encontrada em 1:800 nascidos vivos ${ }^{(1)}$. Considerando-se que a SD é causa de deficiência mental e que esta é fator de risco ao desenvolvimento da linguagem, faz-se necessário o acompanhamento destes indivíduos desde o nascimento, para identificação das manifestações e intervenção o mais precoce possível evitando maiores atrasos no desenvolvimento.

Levando-se em conta que as características da SD, dentre elas a DM, são variáveis importantes para o desenvolvimento global e que existe uma variabilidade de manifestações de linguagem no grupo, sugere-se o seguinte questionamento: "as manifestações clínicas observadas nesse grupo são atípicas, assincrônicas ou típicas quando comparadas ao de crianças sem a síndrome?". Pois, caso sejam típicas, caracterizaria um atraso como citado em muitos trabalhos. Assim sendo, o objetivo do estudo foi caracterizar as habilidades simbólicas de um grupo de crianças com síndrome de Down com idades entre 12 e 36 meses.

\section{MÉTODOS}

O estudo foi aprovado pelo Comitê de Ética em Pesquisa da Faculdade de Medicina de Ribeirão Preto da Universidade de São Paulo (USP), processo no 5973/2007. Os participantes foram selecionados nas Associações de Pais e Amigos dos Excepcionais (APAEs) de dois municípios no interior do estado de São Paulo e em uma Escola de Educação Infantil, com autorização prévia dos responsáveis pelas instituições e posteriormente dos responsáveis pelas crianças.

Participaram da pesquisa 13 crianças no Grupo Síndrome de Down (GSD) e 13 no Grupo Controle (GC). Os critérios para a seleção do GSD foram: diagnóstico de síndrome de Down, frequentar as instituições (APAEs), exclusão de alteração sensorial auditiva e/ou visual que pudessem comprometer o desenvolvimento da linguagem e idade entre 12 e 36 meses. E para o GC os critérios foram: idade entre 12 e 36 meses e desenvolvimento normal (linguagem e cognição).

Para a coleta e análise dos dados utilizamos o item três (desenvolvimento cognitivo) do Protocolo de Observação Comportamental (PROC) dividido em quatro partes: formas de manipulação dos objetos (pontuação máxima de dez pontos), nível de desenvolvimento do simbolismo (pontuação máxima de 20 pontos), nível de organização do brinquedo (pontuação máxima de 20 pontos) e imitação (pontuação máxima de 20 pontos), sendo 70 , a pontuação máxima nesta avaliação ${ }^{(14)}$.

Para obtenção dos dados usamos brinquedos em miniaturas: um celular, um tambor, um chocalho, uma bola pequena com chocalho, uma boneca, um kit com piranha, gloss, lacinho e pente, um babador, uma mamadeira, dois pentes (um vermelho e um amarelo), quatro xícaras, dois shampoos, um carro maior, um conjunto com cinco potes de encaixe, duas panelas, quatro pratos, uma esponja para fazer barba, um barbeador, uma espátula para barbear, um serrote, três ferramentas (chave, alicate, martelo), seis carrinhos mini, quatro colheres, uma tesoura, uma concha, um garfo, quatro panelinhas, uma peneira, uma girafa, um boi, três cavalos, um leão, um burro, quatro porcos, dois gorilas, um urso, um laço, um pente e escova menores, dois potinhos, uma tampa e um pote dispostos em uma caixa plástica organizadora.

Os dados de uma situação planejada de observação e interação com duas pesquisadoras, alunas do quarto ano de Fonoaudiologia da instituição, foram coletados durante 30 minutos com o GSD, e durante 20 minutos com GC. A situação de interação foi filmada, usando filmadora Panasonic ${ }^{\circledR}$ 
NV-VZ185, em fita VHS-C. Os brinquedos permaneceram dentro de uma caixa plástica à disposição do participante que foram estimulados pelos pesquisadores a abrir e brincar. Em seguida, observou-se as atitudes dos participantes frente aos objetos e ao interlocutor. Para o registro das imagens a filmadora permaneceu disposta em tripé e direcionada para o sujeito a uma distância máxima de um metro. As imagens foram digitalizadas por meio dos programas Pinnacle Studio 9.4.3 ou Sony ${ }^{\circledR}$ Vegas 8.0 e gravadas em DVD-RW. Ao analisar as amostras obtidas com GSD foram desconsiderados os cinco minutos iniciais e dez finais de coleta, para evitar efeitos de falta de familiarização com a situação de interação ao início ou cansaço ao final.

As gravações foram assistidas por três juizes: duas alunas do quarto ano de Fonoaudiologia e um docente, em um microcomputador Pentium Intel, utilizando fone de ouvido. Após assistir as fitas, esses juízes registravam os comportamentos observados no protocolo de registro do item três do PROC, comparavam e discutiam as anotações e sempre que necessário retornavam às amostras, para chegar ao resultado final que era anotado na folha de registro oficial para ser arquivada.

Os dados foram analisados do ponto de vista estatístico. Para as comparações entre as idades e entre os grupos utilizamos o modelo de ANOVA ${ }^{(19)}$. Os ajustes dos modelos foram feitos através do software SAS versão $9^{(20)}$.

\section{RESULTADOS}

Para melhor interpretação e compreensão dos resultados os grupos foram subdivididos de acordo com a idade, ou seja, GSD I e GC I crianças de 12 a 24 meses e GSD II e GC II de 25 a 36 meses. Os resultados serão apresentados de acordo com os subitens contemplados no PROC: a) forma de manipulação dos objetos, b) nível do desenvolvimento do simbolismo, c) nível de organização do brinquedo, e d) imitação gestual e sonora.

Quanto à forma de manipulação dos objetos observou-se que o GSD I explorou os objetos de maneira rápida e superficial, por meio de poucas ações e de modo repetitivo, mesmo que sobre dois objetos ao mesmo tempo, ao passo que o GC I apresentou $40 \%$ de explorações superficiais, $20 \%$ repetitivas e $40 \%$ atuação diversificada ao manipular dois objetos ao mesmo tempo relacionando-os. Ao considerar o parâmetro cronológico constatamos que as características dos GSD I e GSD II foram semelhantes às de crianças entre 12 e 24 meses como as do GC I. Salientamos que no GSD II $14 \%$ dos participantes não se interessou pelos objetos e ocorreu $28 \%$ de desistência das atividades frente a obstáculo. Esses fatos sugerem que a idade mental é um parâmetro importante para a análise de habilidades simbólicas.

Ao analisar o nível de desenvolvimento do simbolismo verificamos que o GSD I apresentou $83 \%$ de condutas sensóriomotoras, não apresentou esquemas simbólicos no próprio corpo e nem uso de bonecos ou outros parceiros no brinquedo simbólico quando comparados ao GC I que apresentou uso convencional dos objetos em $100 \%$ da amostra e condutas pré-simbólicas (aplicação dos objetos em outras pessoas), além de fazer uso da linguagem verbal para relatar situações lúdicas. Identificamos também diferença entre o GSD II e o
GC I e II quanto a este item do desenvolvimento do simbolismo. Destacamos que o GC II obteve $87 \%$ de condutas présimbólicas como uso de parceiros no brinquedo simbólico e todos os participantes utilizaram linguagem verbal para relatar a situação lúdica o que não ocorreu com GSD.

No GSD I, 33\% dos participantes não manifestaram reação às solicitações de imitação e gestos/movimentos visíveis no próprio corpo (como derrubar canecas empilhadas, apalpar esponja de banho, andar com o carro, oferecer mamadeira a boneca), enquanto que no GC I todos os participantes reagiram às solicitações. Na faixa etária de 25-36 meses, no GSD II $14 \%$ dos participantes não reagiram às solicitações de imitação e gestos/movimentos visíveis no próprio corpo e $85 \%$ às solicitações de imitação gestos/movimentos visíveis no próprio corpo. Já no GC II $25 \%$ não reagiram às solicitações verbais e $62 \%$ às solicitações de imitação dos gestos, ou seja, o GC II de acordo com sua idade cronológica apresenta menor porcentagem de imitações e maior número de iniciativas de interação com os objetos. A imitação de gestos/movimentos não visíveis no próprio corpo não foi avaliada nesse estudo.

Em relação às imitações sonoras, o GSD I apresentou respostas semelhantes ao GC I, tais como ausência de reação às solicitações e imitação de palavras, e exceto em imitação de onomatopéias, na qual os GC I e II não apresentaram respostas. Notou-se que o GSD II imitou apenas onomatopéias e palavras enquanto que o GC II apresentou imitação de palavras e frases indicando que o GC está de acordo com o esperado para o desenvolvimento normal no aspecto de imitação sonora. A imitação de sílabas não foi avaliada.

Quanto ao nível de organização do brinquedo os participantes do GSD I e II manipularam os objetos sem uma organização dos mesmos de acordo com o que descreve o item três do PROC, ou seja, não organizaram as miniaturas em pequenos grupos, não realizaram agrupamentos dos objetos, não enfileiraram os objetos, não organizaram os objetos de modo a configurar os diversos cômodos da casa e o mesmo ocorreu nos respectivos grupos controles.

$\mathrm{Na}$ Tabela 1 e na Figura 1 observa-se a comparação de média dos escores obtidos com a análise do item três do PROC intra-grupos, ou seja, GSD I com GSD II e GC I com GC II. Assinalamos as manifestações que se diferenciaram quando comparadas de acordo com as idades.

Encontramos diferenças $(\mathrm{p}<0,05)$ comparando os GC I e GC II para as formas de manipulação dos objetos, para o nível de desenvolvimento do simbolismo e para o desempenho geral no PROC, e nos GSD I e GSD II a diferença significativa ocorreu para o nível de desenvolvimento do simbolismo, pois o GSD I apresentou 83\% de condutas sensório-motoras e não pontuou as outras habilidades simbólicas descritas e o GSD II apresentou $71 \%$ de uso convencional dos objetos com início de outras habilidades simbólicas. As médias dos GSD I e GC I estavam abaixo das médias dos GSD II e GC II. Não houve diferença na imitação sonora.

Na Tabela 2 e na Figura 2 observa-se a comparação de média dos escores obtidos com a análise do item três do PROC inter-grupos.

Assim como na comparação entre GSD I e II e GC I e II entre si, não foram encontradas diferenças quanto à imitação 
Tabela 1. Comparação de médias entre as idades em cada grupo

\begin{tabular}{|c|c|c|c|c|c|c|c|}
\hline Variável & Grupo & $\begin{array}{c}\text { Comparação } \\
\text { (anos) }\end{array}$ & Média & $\mathrm{DP}$ & Diferença & IC (95\%) & Valor de $p$ \\
\hline \multirow[t]{4}{*}{ Escore 3a } & Down & $1-2$ & 2,83 & 1,17 & $-0,60$ & $(-2,80 ; 1,61)$ & 0,58 \\
\hline & & $2,1-3$ & 3,43 & 1,72 & & & \\
\hline & Normal & $1-2$ & 6,00 & 3,74 & $-4,00$ & $(-6,26 ;-1,74)$ & $0,01^{*}$ \\
\hline & & $2,1-3$ & 10,00 & 0,00 & & & \\
\hline \multirow[t]{4}{*}{ Escore 3b } & Down & $1-2$ & 0,17 & 0,41 & $-4,98$ & $(-8,78 ;-1,15)$ & $0,01^{*}$ \\
\hline & & $2,1-3$ & 5,14 & 4,74 & & & \\
\hline & Normal & $1-2$ & 7,20 & 2,77 & $-4,68$ & $(-8,59 ;-0,76)$ & $0,02^{*}$ \\
\hline & & $2,1-3$ & 11,88 & 3,27 & & & \\
\hline \multirow[t]{4}{*}{ Escore 3d } & Down & $1-2$ & 3,33 & 3,67 & $-0,24$ & $(-3,90 ; 3,43)$ & 0,89 \\
\hline & & $2,1-3$ & 3,57 & 3,21 & & & \\
\hline & Normal & $1-2$ & 3,00 & 2,74 & $-2,88$ & $(-6,63 ; 0,88)$ & 0,13 \\
\hline & & $2,1-3$ & 5,88 & 3,00 & & & \\
\hline \multirow[t]{4}{*}{ Escore geral } & Down & $1-2$ & 6,33 & 4,41 & $-5,81$ & $(-12,97 ; 1,36)$ & 0,11 \\
\hline & & $2,1-3$ & 12,14 & 7,86 & & & \\
\hline & Normal & $1-2$ & 16,20 & 6,72 & $-11,55$ & $(-18,89 ;-4,21)$ & $0,01^{*}$ \\
\hline & & $2,1-3$ & 27,75 & 5,34 & & & \\
\hline
\end{tabular}

Teste Anova. * Valores significativos $(p<0,05)$

Legenda: DP = desvio-padrão; IC = intervalo de confiança

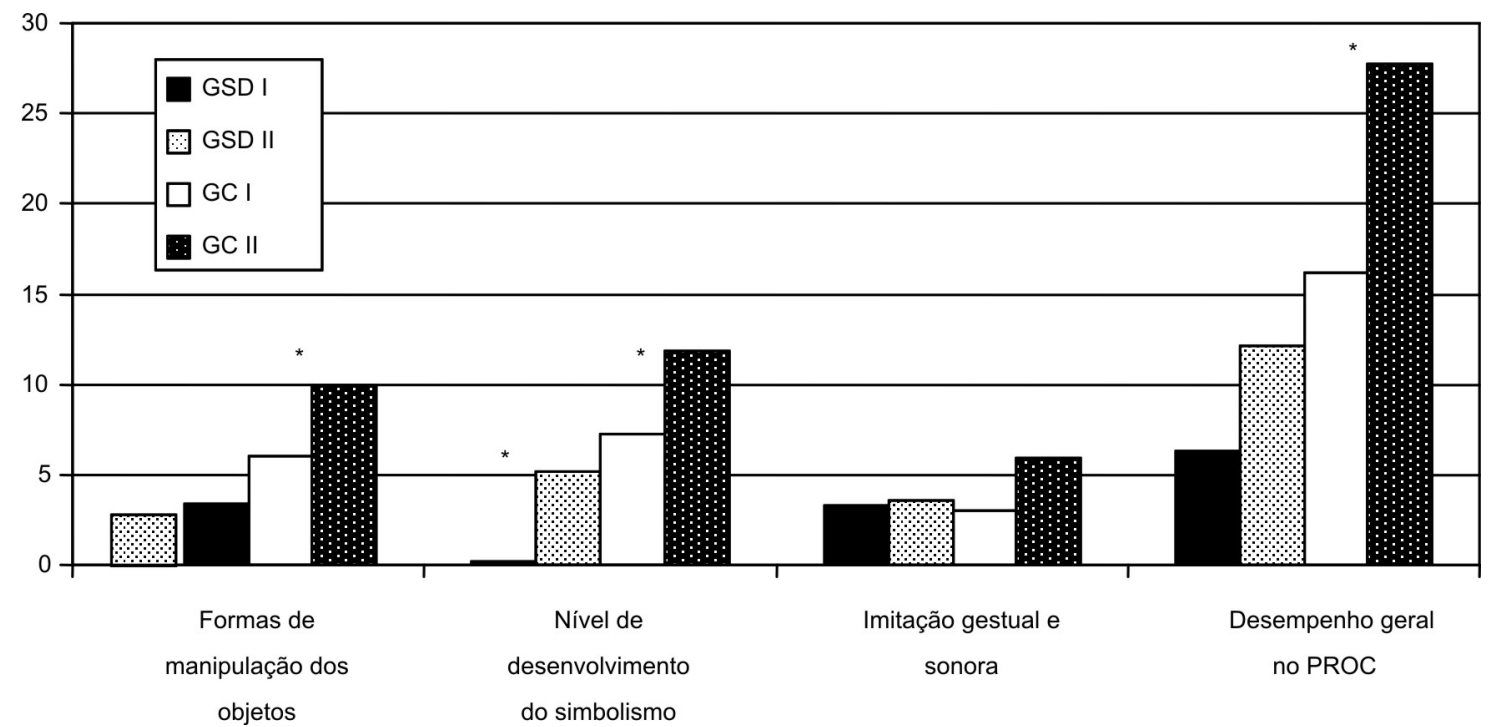

Teste Anova. ${ }^{*}$ Valores significativos $(p<0,05)$

Legenda: GSD I = grupo síndrome de Down I; GSDII = grupo síndrome de Down II; GCI = grupo controle I; GCII = grupo controle II; PROC = protocolo de observação comportamental

Figura 1. Médias dos escores do PROC intra-grupos

sonora e gestual também na comparação entre GSD I e GCI e GSD II e GC II.

\section{DISCUSSÃO}

As crianças dos GSD I e II apresentaram atraso em relação às crianças do GC de ambas as faixas etárias, e como esperado em crianças com síndrome de Down, a brincadeira simbólica estava significantemente atrasada em relação a ambos GC, porém as habilidades presentes eram as mesmas observadas no desenvolvimento normal ${ }^{(9,11,12)}$.

A maneira como a criança manipula os objetos à sua volta pode revelar um atraso ou não na linguagem e outros aspectos do desenvolvimento ${ }^{(13)}$. Verificamos com esta pesquisa que a forma de manipulação dos objetos ocorreu de maneira diferente tanto comparando as médias intra-grupos quanto as médias inter-grupos, ou seja, as crianças do GSD realizaram ações aquém do esperado para a idade cronológica.

Uma amostra de crianças com SD apresentou jogo simbólico aos 22 meses $^{(21)}$, resultado diferente do observado no 
Tabela 2. Comparação de médias entre os grupos em cada idade

\begin{tabular}{|c|c|c|c|c|c|c|c|}
\hline Variável & Grupo (anos) & Comparação & Média & $\mathrm{DP}$ & Diferença & IC (95\%) & Valor de $p$ \\
\hline \multirow[t]{4}{*}{ Escore 3a } & $1-2$ & Down & 2,83 & 1,17 & $-3,17$ & $(-5,57 ;-0,76)$ & $0,01^{*}$ \\
\hline & & Normal & 6,00 & 3,74 & & & \\
\hline & $2,1-3$ & Down & 3,43 & 1,72 & $-6,57$ & $(-8,63 ;-4,52)$ & $0,01^{*}$ \\
\hline & & Normal & 10,00 & 0,00 & & & \\
\hline \multirow[t]{4}{*}{ Escore 3b } & $1-2$ & Down & 0,17 & 0,41 & $-7,03$ & $(-11,19 ;-2,87)$ & $0,01^{*}$ \\
\hline & & Normal & 7,20 & 2,77 & & & \\
\hline & $2,1-3$ & Down & 5,14 & 4,74 & $-6,73$ & $(-10,29 ;-3,18)$ & $0,01^{*}$ \\
\hline & & Normal & 11,88 & 3,27 & & & \\
\hline \multirow[t]{4}{*}{ Escore 3d } & $1-2$ & Down & 3,33 & 3,67 & 0,33 & $(-3,66 ; 4,32)$ & 0,86 \\
\hline & & Normal & 3,00 & 2,74 & & & \\
\hline & $2,1-3$ & Down & 3,57 & 3,21 & $-2,30$ & $(-5,71 ; 1,11)$ & 0,18 \\
\hline & & Normal & 5,88 & 3,00 & & & \\
\hline \multirow[t]{4}{*}{ Escore Geral } & $1-2$ & Down & 6,33 & 4,41 & $-9,87$ & $(-17,67 ;-2,07)$ & $0,02^{*}$ \\
\hline & & Normal & 16,20 & 6,72 & & & \\
\hline & $2,1-3$ & Down & 12,14 & 7,86 & $-15,61$ & $(-22,27 ;-8,94)$ & $0,01^{*}$ \\
\hline & & Normal & 27,75 & 5,34 & & & \\
\hline
\end{tabular}

Teste Anova. * Valores significativos $(p<0,05)$

Legenda: $\mathrm{DP}$ = desvio-padrão; IC = intervalo de confiança

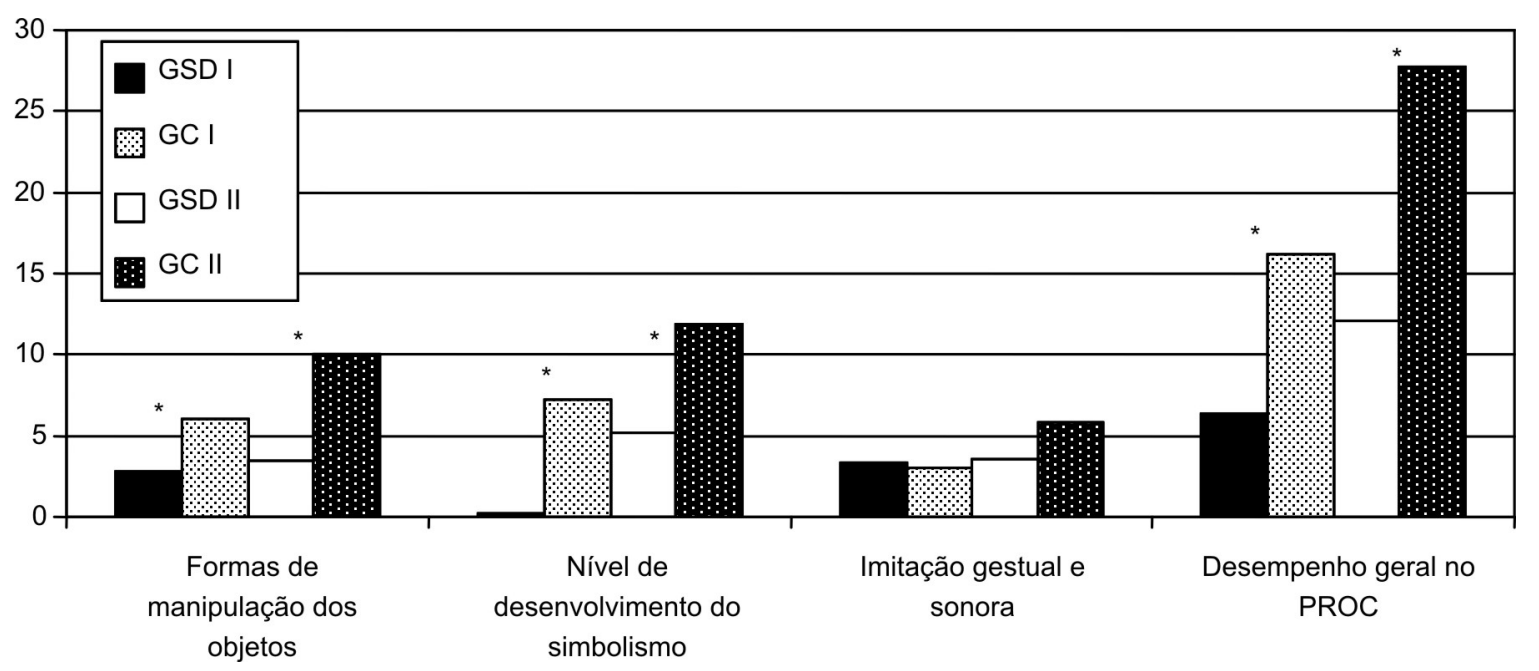

Teste Anova. * Valores significativos $(p<0,05)$

Legenda: GSD I = grupo síndrome de Down I; GSDII = grupo síndrome de Down II; GCI = grupo controle I; GCII = grupo controle II; PROC = protocolo de observação comportamental

Figura 2. Média dos escores do PROC inter-grupos

presente estudo, no qual os sujeitos não apresentaram habilidades simbólicas nessa idade. Por outro lado, os resultados encontrados dão consistência à observação de jogo simbólico pior para crianças com alteração de linguagem quando comparadas a normais $^{(22)}$; nos grupos desta pesquisa observou-se maior ocorrência de explorações sensório-motoras em relação aos GC.

O PROC instrui o examinador para agir como observador das ações das crianças avaliadas. Todavia, optou-se por introduzir solicitações tanto de imitação gestual quanto sonora para facilitar a observação desses comportamentos; há que se considerar que estas porcentagens tenham sido influenciadas pelas atitudes das examinadoras.
Como as crianças com SD apresentam um atraso de todas as áreas de desenvolvimento ${ }^{(23)}$, a observação do grau de simbolismo e das atividades exploratórias que estão diretamente ligados ao desenvolvimento da linguagem tem importantes implicações para ampliar a compreensão sobre a aquisição de linguagem nesse grupo $^{(18)}$.

Em alguns comportamentos analisados as crianças do GSD I e II comportaram-se de modo semelhante às crianças do GC I e II como é o caso da imitação sonora em que não houve diferença estatística nos resultados, porém não foram encontrados na literatura pesquisada dados para comparação.

A atividade lúdica é de extrema importância para o desenvolvimento de crianças normais e com algum comprometimen- 
to, pois interagindo com os brinquedos, com o meio e com outros parceiros a criança começa a estruturar significados para a brincadeira e aplicar diversas ações vividas no passado, construindo um repertório de conhecimento sobre objetos, pessoas e ações ${ }^{(6,13)}$. Durante as interações observou-se que os objetos mais utilizados pelas crianças foram: boneca, mamadeira, potes, panelas, talheres, telefone e bola. E que as atividades de rotina diária, como oferecer mamadeira a boneca, atender telefone, andar de carro foram as mais encontradas indicando a representação das experiências vividas no dia-a-dia, em uma sucessão de rotinas ${ }^{(24)}$.Nesta pesquisa foram excluídos da análise os cinco primeiros minutos da filmagem e os dez últimos, pois inicialmente as crianças tinham que estabelecer interação com a examinadora, e como esse era o primeiro contato com ela, muitas vezes a interação não ocorria de imediato. Além disso, autores afirmaram que qualquer tempo e momento de filmagem podem ser utilizados para análise do perfil comunicativo de indivíduos com síndrome de Down, sem possibilidade de prejuízo da qualidade e da fidedignidade dos dados ${ }^{(25)}$. Ao analisar as filmagens, com duração de 30 minutos, notamos que as crianças mantiveram um tempo de atenção em média de 15 minutos, nos quais conseguimos uma interação com boa qualidade e sem prejuízos para a análise dos resultados.

Como não havia dados da idade mental das crianças dos GSD I e II, optou-se por realizar os mesmos procedimentos com crianças de desenvolvimento normal da linguagem (GC) visando comparar estes grupos de acordo com as idades cronológicas e mostrar dados da aquisição das habilidades simbólicas do GSD em relação ao GC.
Ao comparar-se a idade cronológica constatou-se um atraso significativo, o que confirma a hipótese de atraso do desenvolvimento das habilidades simbólicas nas crianças com SD estudadas.

\section{CONCLUSÃO}

As crianças com SD estudadas estavam explorando os objetos por meio de poucas ações e de modo repetitivo, através de atividades sensório-motoras, sem uma organização dos mesmos, imitando onomatopéias e palavras e gestos/movimentos visíveis no próprio corpo.

Os resultados obtidos ressaltam a importância da avaliação de habilidades simbólicas em crianças com alteração de linguagem, pois as ações da criança sobre os objetos em atividade lúdica podem revelar atrasos no desenvolvimento das funções simbólicas que precedem o desenvolvimento da linguagem.

Em relação às crianças com síndrome de Down é necessário o diagnóstico e intervenção fonoaudiológicos precoces para que suas habilidades sejam potencializadas e algumas dificuldades superadas.

\section{AGRADECIMENTOS}

Agradecemos às instituições, participantes e seus familiares pela disponibilidade, e à Fundação de Amparo à Pesquisa do Estado de São Paulo (FAPESP), pelo apoio concedido para realização dessa pesquisa, sob processo número 2007/07635-0.

\begin{abstract}
Purpose: To characterize the symbolic abilities of a group of children with Down syndrome. Methods: The study included 26 children with ages ranging from 12 to 36 months, divided into two groups: Down syndrome group (DSG) and control group (CG) - children within normal development. The groups were subdivided according to age range: DSG I and CG I comprised children from 12 to 24 months old; DSG II and CG II, children from 25 to 36 months. Data were gathered during a 30- (DSG) or 20-minute (CG) interaction session with the examiner, in a playful situation, according to the proposal of the behavioral observation protocol. Results: The comparison between the control groups showed differences $(\mathrm{p}<0.05)$ in the forms of objects' manipulation, the level of symbolic development, and in overall performance on the protocol. In both Down syndrome groups there was a difference in the level of symbolic development. The between-group comparison according to age groups indicated differences in how children manipulated objects, in the level of symbolic development, and in overall performance on the protocol. The imitation of sounds and gestures did not differ significantly in this study. Conclusion: The results confirmed the hypothesis of delay in the development of symbolic abilities of children with Down syndrome. The evaluation of language and symbolism in a functional context allowed the confrontation of manifestations observed in this group with those described for children within normal language and symbolic development, showing that the level of symbolic development was the best measure for analyzing and monitoring the group.
\end{abstract}

Keywords: Down syndrome; Child language; Symbolism; Child development; Cognition

\title{
REFERÊNCIAS
}

1. Nussbaum RL, Mcinnes RR, Willard HF. Thompson \& Thompson Genética médica. 6a ed. Rio de Janeiro: Guanabara Koogan; 2002. Citogenética clínica: distúrbios dos autossomos e dos cromossomos sexuais; p. 138-41.
2. Silva NLP, Dessen MA. Síndrome de Down: etiologia, caracterização e impacto na família. Interação Psicol. 2002;6(2):167-76.

3. Limongi SCO. Linguagem na síndrome de Down. In: Ferreira LP, BefiLopes DM, Limongi SCO, organizadores. Tratado de fonoaudiologia. 
São Paulo: Roca; 2004. p. 954-66.

4. Gusmão FAF, Tavares EJM, Moreira LMA. Idade materna e síndrome de Down no Nordeste do Brasil. Cad Saúde Pública = Rep Public Health. 2003;19(4):973-8.

5. Mendes DMLF, Moura MLS. Desenvolvimento da brincadeira e linguagem em bebês de 20 meses. Psicol Teor Pesq. 2004;20(3):215-22.

6. Limongi SCO, Mendes AE, Carvalho AMA, Do Val DC, Andrade RV. A relação comunicação não verbal-verbal na síndrome de Down. Rev Soc Bras Fonoaudiol. 2006;11(3):135-41.

7. Silva MPV, Salomão NMR. Interações verbais e não-verbais entre mãescrianças portadoras de Síndrome de Down e entre mães-crianças com desenvolvimento normal. Estud Psicol (Natal). 2002;7(2):311-23.

8. Voivodic MAMA, Storer MRS. O desenvolvimento cognitivo das crianças com síndrome de Down à luz das relações familiares. Psicol Teor Prat. 2002;4(2):31-40.

9. Horstmeier D. Comunicação. In: Pueschel S. Síndrome de Down: guia para pais e educadores. 2a ed. Campinas: Papirus; 1995. p. 239- 65.

10. Chan JB, Iacono T. Gesture and word production in children with Down Syndrome. Augment Altern Commun. 2001;17(2):73-87.

11. Flabiano FC, Limongi SCO. Relação entre os gestos e a linguagem oral em um par de gêmeos com Síndrome de Down. Rev Soc Bras Fonoaudiol. 2006;11(2):116-23.

12. O'Toole C, Chiat S. Symbolic functioning and language development in children with Down syndrome. Int J Lang Commun Disord. 2006;41(2):155-71.

13. Zorzi JL. A intervenção fonoaudiológica nas alterações da linguagem infantil. 2a ed. Rio de Janeiro: Revinter; 2002. p. 3-46.

14. Zorzi JL, Hage SRV. PROC - Protocolo de observação comportamental: avaliação de linguagem e aspectos cognitivos infantis. São José dos Campos: Pulso Editorial; c2004. 93 p.

15. Scheuer CI, Limongi SCO. Distúrbios cognitivos. In: Limongi
SCO. Fonoaudiologia - Informação para a formação:Linguagem: desenvolvimento normal, alterações e distúrbios. Rio de Janeiro: Guanabara Koogan; 2003. p. 139-57.

16. Stefanini S, Caselli MC, Volterra V. Spoken and gestural production in a naming task by young children with Down syndrome. Brain Lang. 2007;101(3):208-21.

17. Andrade RV, Limongi SCO. A emergência da comunicação expressiva na criança com síndrome de Down. Pró-Fono. 2007;19(4):387-92.

18. Domeniconi C, Costa ARA, Souza DG, Rose JC. Responder por exclusão em crianças de 2 a 3 anos em uma situação de brincadeira. Psicol Reflex Crit. 2007;20(2):342-50.

19. Montgomery DC. Design and analysis of experiments. 5th ed. New York: John Wiley; 2001.

20. SAS/STAT® User's Guide, Version 9. [Internet]. Cary, NC, USA: SAS Institute Inc., 2002-2003. [cited 2010 Jul 12]. Available from: http://post. queensu.ca:8080/SASDoc/getDoc/en/statug.hlp/stat_index.htm

21. Limongi SCO, Andrade RV, Lima FAGF, Alabarse VM, Perez VM. Processo terapêutico fonoaudiológico realizado com um par de gêmeos portadores de Síndrome de Down. Pró-Fono. 2000;12(1):24-33.

22. Befi-Lopes DM, Takiuchi N, Araújo K. Avaliação da maturidade simbólica nas alterações do desenvolvimento da linguagem. J Bras Fonoaudiol. 2000;1(3):6-15.

23. Caselli MC, Vicari S, Longobardi E, Lami L, Pizzoli C, Stella G. Gestures and words in early development of children with Down syndrome. J Speech Lang Hear Res. 1998;41(5):1125-35.

24. Corsi CM, Tanaka CK, Uchimura M, Sprocatti R, Rodrigues R, Limongi SCO. Síndrome de Down, desenvolvimento cognitivo e de linguagem: um estudo de investigação. Temas Desenvolv. 1995;5(25):11-6.

25. Porto E, Limongi SCO, Santos IG, Fernandes FDM. Amostra de filmagem e análise da pragmática na síndrome de Down. Pró-Fono. 2007;19(2):159-66. 